\title{
Avaliação do torque passivo de flexão plantar e torque ativo de flexão dorsal em ginastas rítmicas e não atletas
}

CDD. 20.ed. 796.073

796.405

http://dx.doi.org/10.1590/1807-55092014000300371
Natália Batista Albuquerque GOULART*

Caroline Pieta DIAS*

Fernando de Aguiar LEMOS*

João Carlos OLIVA*

Fábio Juner LANFERDINI*

Marco Aurélio VAZ"
*Escola de Educação

Física, Universidade

Federal do Rio Grande

do Sul.

\section{Resumo}

0 treinamento realizado por atletas de elite acarreta demandas funcionais especificas que produzem mudanças nos tendões e nas propriedades musculares. Nas atletas de ginástica rítmica (GR) há uma exigência maior de controle motor em posições extremas de flexão plantar. Esta demanda pode alterar o torque passivo dos flexores plantares bem como influenciar na amplitude de movimento (ADM) de flexão dorsal e torque dos dorsiflexores. No presente estudo foi comparada a ADM de flexão dorsal de GR e meninas não atletas (MNA), e determinada a correlação entre o torque passivo dos flexores plantares (TPFP) e o torque ativo dos flexores dorsais (TAFD). 0 estudo incluiu 10 GR e 10 MNA. A ADM de flexão dorsal foi medida com um goniômetro. 0 TPFP e TAFD foram avaliados por meio de um dinamômetro isocinético em cinco ângulos articulares $\left(20^{\circ}\right.$, $10^{\circ}, 0^{\circ},-10^{\circ}$ e $-20^{\circ}$ ). 0 teste $T$ para amostras independentes foi utilizado para a comparação entre os grupos $(\alpha=0,05)$. 0 coeficiente de correlação de Pearson indicou uma correlação negativa alta e moderada entre 0 TPFP e TAFD para as GR e NA, respectivamente. GR apresentaram menor ADM de flexão dorsal comparadas às MNA. 0 TPFP foi significativamente maior nas GR comparado as MNA. Por outro lado, o TAFD foi maior nas MNA. A correlação do TPFP com o TAFD foi de $r=-0,82 ; p<0,01$ e $r=-0,68 ; p<0,01$ para GR e MNA, respectivamente. Os resultados sugerem que a alta demanda mecânica nos flexores plantares na GR aumenta o TPFP que pode influenciar negativamente na ADM dos flexores dorsais e no TAFD.

Palavras-chave: Ginástica; Tornozelo; Torque.

\section{Introdução}

A demanda mecânica decorrente do treinamento promove mudanças na estrutura muscular e tendínea, influenciando a capacidade de geração de força dos músculos que agem sobre uma articulação ${ }^{1}$. Em grande parte das modalidades, os grupos musculares agonistas, responsáveis pela execução de movimentos específicos, apresentam maior excursão e desenvolvimento de força, comparados aos antagonistas ${ }^{2}$.

Bailarinas clássicas, por exemplo, apresentam maior força e amplitude de movimento (ADM) nos flexores plantares do que nos flexores dorsais devido, principalmente, ao maior número de exercícios executados em "meia ponta" . Semelhante ao Balé Clássico, a Ginástica Rítmica (GR) é uma modalidade esportiva caracterizada pela grande amplitude de movimento, sendo que muitos exercícios são realizados em máxima amplitude de flexão plantar (FP), exigindo alto grau de equilíbrio e coordenação em "meia ponta". Essa maior exigência sobre os flexores plantares deve gerar alterações na capacidade de produção de força e na flexibilidade do tornozelo destas atletas. Em um estudo ${ }^{5}$ com atletas juvenis de GR foi observado menor ADM de flexão dorsal (FD) nas ginastas comparadas a valores normativos para indivíduos não atletas, podendo estar relacionado ao encurtamento e consequente dificuldade de alongamento dos flexores plantares. De acordo com a literatura, a reduçáo da amplitude de FD está relacionada à maior rigidez e reduçâo da complacência 
do Tendão Calcâneo (TC), ocasionada por maior sobrecarga mecânica imposta de maneira crônica sobre este tecido, aumentando o torque passivo durante o deslocamento articular em direção à $\mathrm{FD}^{6}$. A literatura tem demonstrado a alta contribuição do torque passivo de FP (TPFP) na produção de força máxima em $\mathrm{FP}^{7}$. Entretanto, não foram encontrados estudos que tenham avaliado a influência da força passiva dos flexores plantares na força máxima da musculatura antagonista.

Apesar de, em geral, a maioria dos exercícios na GR serem em FP, os movimentos em FD também são importantes, como nos giros e equilíbrios denominados "cossacos", nas rotaçóes com o

\section{Método}

\section{Amostra}

A amostra foi selecionada de forma intencional e composta por 20 meninas, sendo 10 ginastas rítmicas de alto rendimento $(12,4 \pm 1,35$ anos; $152 \pm 0,04$ cm; 40,56 $\pm 5,92 \mathrm{~kg}$ ) com no mínimo cinco anos de prática no esporte, e $10 \mathrm{MNA}(12,0 \pm 1,33$ anos; 151 $\pm 0,04 \mathrm{~cm} ; 39,84 \pm 5,89 \mathrm{~kg})$. Todos os procedimentos foram cuidadosamente explicados para os pais e/ou responsável de cada participante. Os mesmos assinaram um Termo de Consentimento e uma cópia foi entregue ao responsável. Este estudo foi aprovado pelo Comitê de Ética em Pesquisa da Universidade Federal do Rio Grande do Sul (Parecer no. 2008167).

\section{Procedimentos}

Para parear os grupos pela idade biológica, foi aplicado o protocolo de auto-avaliação proposto por TANNER ${ }^{8}$. Em uma sala reservada, foram apresentados às participantes desenhos dos diferentes estágios de desenvolvimento para as duas características sexuais secundárias (no caso das meninas, o desenvolvimento das mamas e presença de pêlos pubianos). A partir da análise dos desenhos, cada criança indicou na folha as suas características físicas que foram utilizadas para determinar o estágio maturacional. Este procedimento foi devidamente realizado por uma das pesquisadoras do estudo. Todas as meninas foram classificadas como púberes.

A ADM de FD foi medida no membro inferior dominante por meio de um goniômetro universal plástico com as meninas sentadas, mantendo o joelho estendido. Primeiramente, a partir da posição calcanhar no solo, bem como na chegada após os saltos (iniciando pelo amortecimento do movimento a partir do primeiro contato da regiāo anterior do pé no solo). Além disso, a literatura demonstra uma alta prevalência de lesões no tornozelo em ginastas, sendo que o desequilíbrio de força e flexibilidade entre as musculaturas antagonistas estáo entre os fatores de risco para lesôes². Dessa forma, também se torna importante a investigaçáo da capacidade de produçáo de força dos flexores dorsais neste grupo de atletas. O objetivo deste estudo foi comparar a influência do TPFP sobre o torque ativo de FD (TAFD) entre atletas de GR e meninas não atletas (MNA).

neutra $\left(0^{\circ}\right)$ foi solicitado às participantes que realizassem a FD de forma ativa, procurando levar a articulação do tornozelo até o alongamento máximo. Para a avaliação da ADM passiva, também partindo do ângulo de $0^{\circ}$, o avaliador moveu lentamente o tornozelo até a amplitude de FD máxima suportada pela menina. Cada medida era realizada três vezes e contabilizado o valor médio.

Para determinação dos valores de TPFP e TAFD, as meninas foram posicionadas sentadas na cadeira de um dinamômetro isocinético (Biodex Medical System, Shirley - NY, EUA), com o pé preso à prancha de fixaçáo na posição neutra por meio de faixas de velcro $\left(90^{\circ}\right.$, tíbia paralela ao solo), com o joelho mantido estendido $\left(0^{\circ}\right)$ de modo que o eixo de rotação do tornozelo estivesse alinhado com o eixo de rotação do aparelho. O quadril foi mantido flexionado em $70^{\circ}$ para evitar o uso da musculatura extensora do quadril na geraçáo de força no tornozelo. O membro inferior foi fixado por faixas de velcro na cadeira do dinamômetro, com os membros superiores mantidos livres.

Primeiramente, o TPFP foi avaliado em cinco configuraçôes articulares diferentes na seguinte ordem: $20^{\circ}, 10^{\circ}, 0^{\circ},-10^{\circ}$ e $-20^{\circ}$, por um período de 20 segundos, sendo os ângulos negativos representativos da FD. No intervalo entre cada ângulo, o tornozelo era posicionado no ângulo de $30^{\circ}$, semelhante ao estudo de KaWAKAMI et al. ${ }^{6}$ Estes ângulos foram escolhidos para não proporcionar desconforto pelo alongamento muscular e também para evitar o deslocamento do calcanhar na prancha de fixação. Durante a avaliação, o tornozelo foi movido de forma passiva automaticamente pelo dinamometro até o ângulo articular 
selecionado, em que era registrado o torque passivo. Foi solicitado às participantes que mantivessem a musculatura relaxada durante o registro do torque, sem a execução de qualquer movimento. Simultaneamente, a linha do torque era acompanhada no monitor do dinamometro ("feedback" visual) de modo a monitorar qualquer contração muscular ${ }^{6}$. Se alguma alteração na linha do torque fosse observada, o teste era interrompido e imediatamente repetido.

Após o período de descanso de 10 minutos, o torque máximo isométrico de FD foi avaliado nos mesmos ângulos articulares citados anteriormente, de maneira randomizada. Para o registro do TAFD, as meninas foram instruídas a produzir força o mais rápido possível até atingir a sua capacidade máxima durante cinco segundos. Entre cada contração, foi observado o intervalo de dois minutos para minimizar os efeitos da fadiga. O teste sempre foi repetido caso fossem verificadas as seguintes situaçóes: a) quando o avaliador ou o próprio sujeito percebeu que não foi obtida uma contração máxima, ou b) quando a contração não foi sustentada por pelo menos um segundo antes de

\section{Resultados}

A ADM de FD ativa e passiva foi significativamente menor nas atletas de GR, comparadas às MNA, respectivamente (Ativa: $2,50 \pm 0,70^{\circ} ; 9,80 \pm 2,57^{\circ}$, $\mathrm{t}(18)=$ $-8,65[-9,07 ;-5,52] ; \mathrm{p}=0,04$ e Passiva: 7,30 $\pm 0,94^{\circ}$; $\left.14,6 \pm 3,13^{\circ}, \mathrm{t}(18)=-7,05[-9,47 ;-5,12] ; \mathrm{p}=0,01\right)$.

Os dados referentes ao TPFP dos dois grupos nos cinco ângulos articulares são apresentados na FIGURA 1A. OTPFP foi significativamente maior nas atletas de GR em todos os ângulos articulares, comparadas às MNA \{20\%: $\mathrm{t}(18)=5,52$ [6,07; 2,72]; $\mathrm{p}<0,01 ; 10^{\circ}: \mathrm{t}(18)=6,97[7,02 ; 3,77] ; \mathrm{p}<0,01$; $0^{\circ}: \mathrm{t}(18)=6,45[7,55 ; 3,84] ; \mathrm{p}<0,01 ;-10^{\circ}: \mathrm{t}(18)$ $=8,50[8,72 ; 5,27] ; \mathrm{p}<0,00 ;-20^{\circ}: \mathrm{t}(18)=10,44$ $[10,45 ; 6,94] ; \mathrm{p}<0,00\}$.

Os dados referentes ao TAFD dos dois grupos nos cinco ângulos articulares são apresentados relaxar, assim como proposto em outros estudos da literatura ${ }^{9}$. Somente os valores de torque ativo foram normalizados pela massa corporal de cada menina.

\section{Análise estatística}

A homogeneidade e a normalidade dos dados foram verificadas por meio dos testes Levene e Shapiro-Wilk, respectivamente. Análise descritiva foi utilizada para caracterizar a amostra. O teste $T$ independente foi utilizado na comparação das variáveis ADM de FD, TPFP e TAFD entre os grupos. Para verificar o grau de associação entre as variáveis TPFP e TAFD foi utilizada a correlação produto-momento de Pearson. A força de correlação foi determinada a partir dos seguintes critérios: $\mathrm{r}=$ 0,90-1,00 (muito alta), 0,70-0,89 (alta), 0,50-0,69 (moderada), 0,26-0,49 (baixa), e 0,00-0,25 (correlação nula $)^{7}$. O coeficiente de determinação $\left(r^{2}\right)$ foi calculado. Todos os testes foram realizados no pacote estatístico SPSS 20.0 for Windows (Chicago, EUA). $\mathrm{O}$ nível de significância adotado foi $\alpha=0,05$.

na FIGURA 1B. O TAFD foi significativamente maior nas MNA em todos os ângulos articulares, comparadas às atletas $\left\{20^{\circ}: \mathrm{t}(18)=-7,40[-0,14\right.$; $-0,25] ; \mathrm{p}<0,01 ; 10^{\circ}: \mathrm{t}(18)=-6,83[-0,12 ;-0,24]$; $\mathrm{p}<0,01 ; 0^{\circ}: \mathrm{t}(18)=-5,06[-0,08 ;-0,20] ; \mathrm{p}<0,01$; $-10^{\circ}: \mathrm{t}(18)=-3,82[-0,04 ;-0,16] ; \mathrm{p}<0,01 ;-20^{\circ}$ : $\mathrm{t}(18)=-3,01[-0,02 ;-0,15] ; \mathrm{p}<0,01\}$.

$\mathrm{O}$ grupo de ginastas rítmicas apresentou alta correlação negativa e significativa entre o TPFP e o TAFD (r: -0,82; p < 0,01). O coeficiente de determinação $r^{2}$ indicou que $67 \%$ da variância do TAFD foi atribuída ao TPFP (FIGURA 2A). Para o grupo de MNA, o TPFP apresentou uma moderada correlação significativa com o TAFD (r: -0,68; $\mathrm{p}<$ $0,01)$, com coeficiente de determinação $\mathrm{r}^{2}$ de $46 \%$ (FIGURA 2B). 
* indicam diferença significativa entre os grupos $(p<0,05)$

Todos os valores de torque são expressos em média e erro padrão.
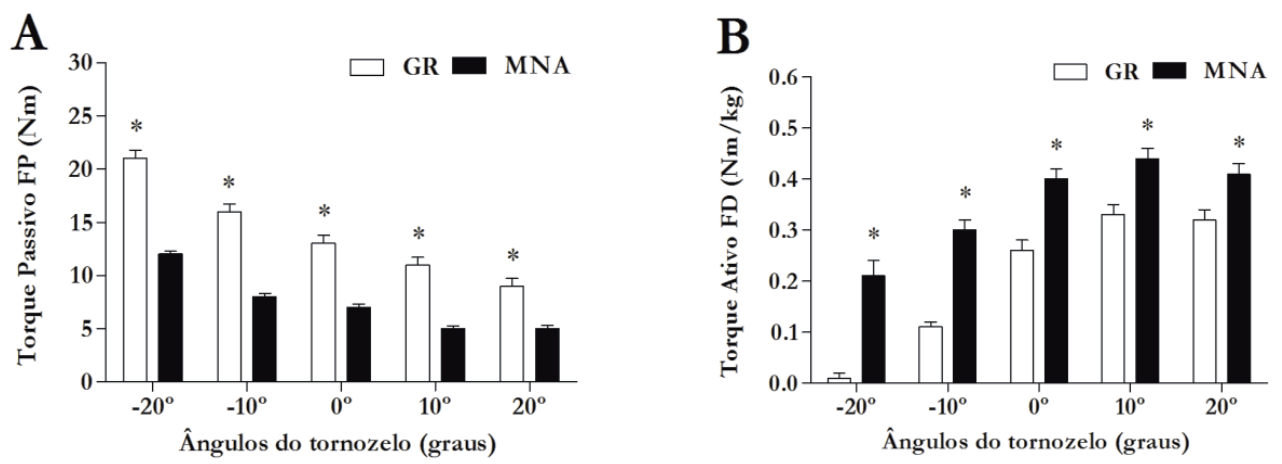

FIGURA 1 - (A) Comparação do torque passivo (Nm) de flexão plantar (FP) em cinco ângulos articulares $\left(^{\circ}\right)$ entre atletas de Ginástica Rítmica (GR) e meninas não atletas (MNA). (B) Comparação do torque ativo $(\mathrm{Nm} / \mathrm{kg})$ de flexão dorsal (FD) em cinco ângulos articulares $\left(^{\circ}\right)$ entre atletas de Ginástica Rítmica (GR) e meninas não atletas (MNA).
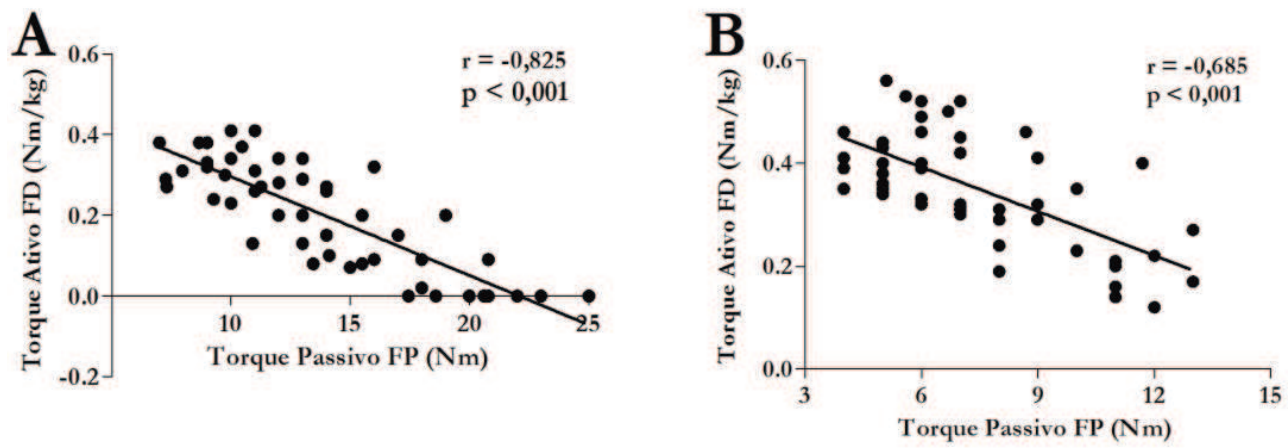

FIGURA 2 - (A) Correlação entre os valores médios de torque passivo de flexão plantar (FP) e os valores médios de torque ativo de flexão dorsal (FD) nas atletas de Ginástica Rítmica. (B) Correlação entre os valores médios de torque passivo de flexão plantar (FP) e os valores médios de torque ativo de flexão dorsal (FD) no grupo de meninas não atletas.

\section{Discussão}

Os resultados relacionados às características antropométricas e estágio maturacional não demonstraram diferenças significativas entre os grupos. De acordo com a literatura, a capacidade de produção de força muscular varia conforme o estágio de maturaçáo, devido, principalmente, a fatores hormonais ${ }^{10}$. Neste estudo, a influência da maturação na produção de força parece ter sido controlada, uma vez que ambos os grupos apresentavam o mesmo estágio maturacional.

O grupo de atletas de GR apresentou menor amplitude de flexão dorsal comparadas às MNA. Este resultado pode estar associado ao maior encurtamento dos flexores plantares e a maior dificuldade de alongamento destes músculos nas ginastas ${ }^{3}$. Entretanto, mesmo no Balé Clássico e na ginástica rítmica, em que a maioria dos exercícios ocorre em flexão plantar máxima, as amplitudes em dorsiflexão também são importantes. No Balé Clássico podem-se citar, principalmente, os movimentos "demi-plie" e "grand-plie", caracterizados pela grande amplitude de flexão do joelho e flexão dorsal. Nas ginastas rítmicas, os exercícios em dorsiflexão são semelhantes aos do Balé Clássico e necessários, principalmente nos equilíbrios e giros denominados "cossacos". Além disso, a musculatura flexora dorsal é muito importante na estabilização do tornozelo no momento do apoio do pé no solo após um salto, sendo que este movimento apresenta uma grande prevalência de entorses de tornozelo em ginastas ${ }^{2}$. A menor ADM de flexão dorsal nas atletas representa um déficit que pode interferir no desempenho desportivo, tornando importante o trabalho de flexibilidade do tornozelo em termos de 
dorsiflexão que poderá tanto produzir a melhora na execução dos movimentos específicos, quanto atuar na prevenção de lesóes no tornozelo ${ }^{11-12}$.

Além disso, o TPFP foi significativamente maior nas atletas de GR comparado às MNA. De acordo com a literatura, o torque passivo produzido durante um alongamento em direção à dorsiflexão também é um fator limitante da ADM e pode ser resultante de mecanismos neurais (reflexo miotático), intrínsecos musculares (miofibrilas e envoltórios musculares) e tendíneos ${ }^{13}$. Está bem evidenciado que a maior sobrecarga mecânica decorrente do treinamento sobre os flexores plantares determina a maior rigidez do TC e mudanças no comprimento muscular, determinando o aumento no torque passivo ${ }^{1,6}$.

A literatura tem demonstrado que um maior TPFP contribui na geração de força máxima durante a flexão plantar ${ }^{7}$. Entretanto, neste estudo foi evidenciada uma interferência negativa deste torque passivo sob a força máxima da musculatura dorsiflexora, uma vez que o alongamento da unidade musculotendínea dos flexores plantares realiza uma força de resistência contrária ao movimento de FD.

Em geral, a habilidade dos extensores em gerar força isométrica é menor quando comparada aos flexores, devido a fatores como diferença no conteúdo de colágeno do tecido tendíneo e também ao volume muscular ${ }^{14}$. De maneira semelhante, a capacidade dos dorsiflexores em gerar força máxima isométrica é menor, comparada à unidade musculotendínea do tríceps sural ${ }^{15-16}$. Essa diferença aumenta em resposta a maior sobrecarga sobre os flexores plantares, podendo gerar desequilíbrio nas relações de geração de força e flexibilidade entre flexores plantares e dorsais.

Dessa forma, o ganho de ADM em flexão dorsal, bem como a inserção de métodos de fortalecimento desta musculatura na rotina de treinamento da ginástica rítmica, pode minimizar a resistência passiva dos flexores plantares sobre a produção de força ativa dos flexores dorsais a qual apresenta uma grande importância tanto para a estabilizaçáo do tornozelo, quanto para a execução de movimentos específicos em dorsiflexão.

\begin{abstract}
Evaluation of passive plantarflexion torque and active dorsiflexion torque in rhythmic gymnasts and nonathletes

The training performed by elite athletes leads to specific functional demands, producing changes on tendon and muscle properties. In the rhythmic gymnastics $(R G)$ athletes there is a higher motor control demand in extreme plantarflexed positions. This functional demand can change the passive torque produced by the plantarflexors, as well as influence the dorsiflexion range of motion (ROM) and maximal dorsiflexion torque. We compared the dorsiflexor ROM and the influence of the passive plantarflexion torque (PPT) on the active dorsiflexion torque (ADT) between RG and nonathletes (NA) girls. The study included 10 $\mathrm{RG}$ and $10 \mathrm{NA}$. The dorsiflexor ROM was measured with a goniometer. The PPT and ADT were evaluated through isokinetic dynamometry at five ankle joint angles $\left(20^{\circ}, 10^{\circ}, 0^{\circ},-10^{\circ},-20^{\circ}\right)$. Independent $\mathrm{T}$ test was used to comparison between groups $(\alpha=0.05)$. Pearson correlation coefficients indicated a high and moderate negative correlation between PPT and ADT for RG and NA, respectively. RG had a smaller dorsiflexion ROM than NA. The PPT was significantly higher in RG compared NA. In contrast, the ADT was higher in NA. The PPT correlation to ADT was $r=-0.82 ; p<0.01$ and $r=-0.68 ; p<0.01$, for $R G$ and $N A$, respectively. Results suggest that higher mechanical loading on plantarflexors in the RG increase the PPT that influence negatively in the dorsiflexor ROM and ADT.
\end{abstract}

KEY WORDS: Gymnastics; Ankle; Torque. 


\section{Referências}

1. Kubo K, Kanehisa H, Kawakami Y, et al. Elastic properties of muscle-tendon complex in long-distance runners. Eur J Appl Physiol. 2000;81:181-7.

2. Zetaruk MN, Violan M, Zurakowski D, et al. Injuries and training recommendations in elite rhythmic gymnastics". Medic del'esport. 2006;151:100-6.

3. Frasson VB, Rassier DE, Herzog W, et al. Dorsiflexor and plantarflexor torque-angle and torque-velocity relationships of classical ballet dancers and volleyball players. Rev Bras Biomec. 2007;8:3-6.

4. Federação Internacional de Ginástica. Código de Pontuação de Ginástica Rítmica 2009-2012. [Lausanne]: FIG; 2009.

5. Goulart NBA. Plasticidade neuromuscular dos flexores plantares em atletas de ginástica rítmica e ginástica artística [monografia]. Porto Alegre: Universidade Federal do Rio Grande do Sul, Escola de Educação Física; 2010.

6. Kawakami Y, Kanehisa H, Fukunaga T. The relationship between passive ankle plantar flexion joint torque and gastrocnemius muscle and Achilles tendon stiffness: implications for flexibility. J Orth Spor Phys Ther. 2008;38:269-76.

7. Gajdosik RL. Relationship between passive properties of the calf muscles and plantarflexion concentric isokinetic torque characteristics. Eur J Appl Physiol. 2002;87:220-7.

8. Matsudo SMM, Matsudo VKR. Validade de auto-avaliação na determinação da maturação sexual. Rev Bras Ciênc Mov. 1991;5:18-35.

9. Herzog W, Guimaraes AC, Anton MG, et al. Moment-length relations of rectus femoris muscles of speed skaters/cyclists and runners. Med Sci Sport Exerc. 1991;23:1289-96.

10. Gallahue D, Osmun J. Compreendendo o denvolvimento motor. São Paulo: Phorte; 2001.

11. Fração VB, Vaz MA, Ragasson CAP, et al. Efeito do treinamento na aptidáo física da bailarina clássica. Movimento. 1999;5:3-15.

12. Hamilton WG, Hamilton LH, Marshall P, et al. A profile of the musculoskeletal characteristics of elite professional ballet dancers. Am J Sports Med. 1992;20:267-73.

13. Abellaneda S, Guissard N, Duchateau J. The relative lengthening of the myotendinous structures in the medial gastrocnemius during passive stretching differs among individuals. J Appl Physiol. 2009;106:169-77.

14. Komi PV. Força e potência no esporte. Porto Alegre: Artmed; 2006.

15. Calmels PM, Nellen M, van der Borne I, et al. Concentric and eccentric isokinetic assessment of flexor-extensor torque ratios at the hip, knee, and ankle in a sample population of healthy subjects. Arch Phys Med Rehabil. 1997;78:1224-30.

16. Poulis S, Poulis I, Soames RW. Torque characteristics of the ankle plantarflexors and dorsiflexors during eccentric and concentric contraction in healthy young males. Isok Exerc Sci. 2000;8:195-202.

\section{Agradecimentos}

À FAPERGS e ao CNPQ pelo apoio financeiro, às participantes, às técnicas das equipes de GR do Clube Grêmio Náutico União e Colégio Salesiano Dom Bosco, e à Federação Riograndense de Ginástica pela colaboração no desenvolvimento deste trabalho.

$$
\begin{array}{r}
\text { ENDEREÇO } \\
\text { Fábio Juner Lanferdini } \\
\text { Laboratório de Pesquisa do Exercício } \\
\text { Escola de Educação Física } \\
\text { Universidade Federal do Rio Grande do Sul } \\
\text { R. Felizardo, 750 - Jd. Botânico } \\
\text { 90690-200 - Porto Alegre - RS - BRASIL } \\
\text { e-mail: fabiolanferdini@gmail.com }
\end{array}
$$

Recebido para publicação: 09/05/2013

Revisado: 30/01/2014

Aceito: 10/06/2014 